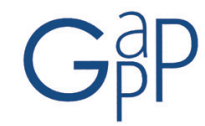

GESTIÓN Y ANÁLISIS DE POLÍTICAS PÚBLICAS, Nueva Época, n 10 julio-diciembre 2013 ISSN: 1989-8991

DOI: http://dx.doi.org/10.24965/gapp.voi10.10117

\title{
El impacto de la crisis en el empleo público local: el caso del área metropolitana de Valencia ${ }^{1}$
}

\author{
J. Javier Cuenca Cervera \\ Universidad de Valencia \\ j.javier.cuenca@uv.es \\ Joaquín Martín Cubas \\ Universidad de Valencia \\ joaquin.martin@uv.es \\ Antonio Montiel Márquez \\ Universidad de Valencia \\ Antonio.Montiel@uv.es \\ Cristina Jiménez Tomás \\ Universidad de Valencia \\ crisjito@alumni.uv.es \\ María José Alonso Orsini \\ Universidad de Valencia \\ majoaor@alumni.uv.es
}

Recibido: 20 de octubre de 2013 Aceptado: 4 de enero de 2014

\begin{abstract}
Resumen
Los ajustes derivados de actual proceso de consolidación fiscal en que se hallan las Administraciones Públicas españolas han golpeado con fuerza a un entramado institucional débil: el empleo público de los gobiernos locales de nuestro país, explicable tanto por la fragilidad sobre el terreno del sistema de mérito, como por la falta de aplicación efectiva de mecanismos de gestión. En este contexto y transcurridos ya cinco años de crisis económica se trata de dar cuenta, mediante datos agregados y de un estudio realizado en el marco del proyecto SAIS-AMV ("sostenibilidad social y ambiental en espacios metropolitanos: el caso del área metropolitana de Valencia"), de qué modo, mediante qué estrategias y sobre qué políticas públicas se han producido los ajustes en el ámbito de los recursos humanos, al mismo tiempo que se ensaya una explicación sobre la senda de ajuste seguida.
\end{abstract}

Palabras clave

Gobiernos locales, recursos humanos, áreas metropolitanas, crisis, nuevo institucionalismo

\section{The impact of the crisis in the employment in local governments: the case of the metropolitan area in \\ Valencia}

\begin{abstract}
The current adjustments from fiscal consolidation that are the Spanish government have been beaten to force a weak institutional framework: public employment of local governments in our country, explained both by the lack of effective institutionalization of a system merit as well as weak of management mechanisms. In this context, and after five years and economic crisis is to account using aggregate data from a study conducted in the framework of the SAIS-AMV ("social and environmental sustainability in metropolitan areas: the case of the metropolitan area of Valencia") how, through what strategies and what public policy adjustments have occurred in the field of human resources at the same time it attempts to explain on the adjustment path.

Keywords

Local government, human resources management, metropolitan areas, crisis, new institutionalism

1 Este trabajo se ha realizado en el marco del Proyecto de investigación "Sostenibilidad ambiental y social en espacios metropolitanos: el caso del área metropolitana de Valencia" (referencia CSO2010-20481-subprograma 6E06) financiado por el Ministerio de Ciencia e Innovación (Plan Nacional I+D+i 2008-2011). Asimismo, una versión previa del mismo fue presentada en el XI Congreso de la Asociación Española de Ciencia Política y de la Administración, celebrado en Sevilla entre los días 18 a 20 de septiembre de 2013, en el Grupo de Trabajo 5.3, "La transformación de la gestión de recursos humanos en el sector público: ajustando dimensiones o generando nuevos modelos" coordinado por los profesores Ramón Bouzas y Miquel Salvador, a quienes agradecemos sus atinadas observaciones, así como a los evaluadores anónimos de la Revista.
\end{abstract}




\section{INTRODUCCIÓN: OBJETO DE LA INVESTIGACIÓN. MARCO TEÓRICO Y METODOLÓGICO}

En el prólogo a la edición española Stiglitz (2012) se pronuncia sin ambages por calificar como una Depresión el efecto en nuestro país de la crisis económica, ya denominada como "Gran Recesión" por los historiadores económicos (Llopis y Maluquer, 2012), un calificativo que la acerca más a la crisis de 1929 que a las de 19730 1979. En este contexto, transcurridos ya cinco años de crisis empiezan a adivinarse algunas tendencias. Una de ellas es la que Beck (2012:43) denomina como "escenario Carl Schmitt", una suerte de estado de excepción transnacional que supone para los países deudores una cierta suspensión de soberanía. Y esa excepcionalidad ha sido palpable en nuestro país: en mayo de 2010 (medidas extraordinarias de reducción del déficit); en 2011 (reforma del artículo 135 de la Constitución) y en 2012 (Ley Orgánica 2/2012, de 27 de abril), que han dado carta de naturaleza a conceptos como el de estabilidad presupuestaria o el de sostenibilidad financiera. Principios llamados a alterar sensiblemente el panorama de nuestro sector público.

$Y$ es que efectivamente, avanzado 2013 puede afirmarse que la Administración que salga de esta crisis, al menos la española, será radicalmente diferente (Ramió, 2011). También su Función Pública (Fuentetaja, 2012:74). Como han destacado autores como Jiménez Asensio (2012:40) en estos momentos nos hallamos todavía bajo los efectos mediatos de un paquete de ajustes (en especial los reales decretos-leyes 20/2011, de 30 de diciembre y 20/2012, de 13 de julio) que han establecido un marco normativo contingente, en especial este último, superpuesto a unas reglas ordinarias de gestión del personal público formalmente no derogadas, pero cuyo impacto sobre la totalidad del sector público está aún por determinar.

A este respecto y en términos de empleo público, sistémicos, hay que tener en cuenta que tales medidas recaen sobre un marco institucional caracterizado por rasgos que administrativistas como Sánchez Morón (2012:250) o Palomar-Olmeda (2012:112) han descrito como de rigidez o exceso de politización. Un efecto del marco normativo promulgado en 2011 y 2012 ha sido apartar el tímido intento reformista que supuso el Estatuto Básico del Empleado Público (EBEP) de 2007, ya que esta ley ha sido literalmente sepultada por esta avalancha de normas "anticrisis", en donde una de sus innovaciones, la creación de la institución "Empleo Público" como paraguas conceptual y material bajo el que cobijar todas las relaciones de empleo en la Administración, con independencia de su régimen jurídico ha sido laminado por el "rancio corporativismo" de las normas "de Función Pública" (Jiménez Asensio, 2013:22). En términos jurídico-formales este parece ser uno de los primeros efectos del periodo: el resurgimiento de la vetusta distinción entre empleo público "laboral” y empleo público "funcionarial", en donde la distinción fundamental entre ambos, la inamovilidad, da lugar a una estructura de incentivos que, como se verá, influirá parcialmente en el comportamiento de los actores que operan en este entorno institucional.

Pero con ser relevantes, las cualitativas no son las únicas tendencias que empiezan a perfilarse, ya que las magnitudes empiezan a adquirir importancia. En especial en un ámbito determinado, el "Empleo Público Local" (EPL), hasta ahora remiso a tales tendencias. A la luz de las normas contingentes no nos hallaríamos ante reformas, sino ante lo que Jiménez Asensio ha calificado como medidas de "ajuste sin reforma" que operan sobre unos colectivos laborales concretos, denominados por este autor como "círculos de vulnerabilidad": el personal no permanente (2011:45)2. Esta sería la finalidad del trabajo, dar respuesta a preguntas de investigación como las siguientes: ¿Qué tipos de medidas de ajuste han adoptado los ayuntamientos de nuestro país? ¿Sobre qué ámbitos de políticas públicas? Al respecto se plantea la hipótesis de que los ayuntamientos habrían focalizado los ajustes sobre el personal temporal, esto es, funcionarios interinos y laborales temporales, sin priorizar políticas sectoriales, basándose exclusivamente en su facilidad de extinción y sin emplear marcos de planificación de recursos humanos a medio o largo plazo.

Para dar respuesta a los interrogantes planteados, la metodología utilizada sigue la recomendación que Peters (2003) efectuara para los científicos sociales que investigan el sector público: mediante una doble aproximación. Atendiendo en primer lugar a la perspectiva estructural, los efectos agregados (Lago, 2008:18). Pero al tiempo hay que tener en cuenta el efecto independiente de la acción social en ese entramado institucional. Por ello,

2 El calificativo de "ajuste sin reforma" vendría dado no solamente por las medidas a las que se ha hecho referencia en los citados reales-decretos ley 20/2011 y 20/2012, ambos con una clara finalidad de contención del déficit y de estabilidad presupuestaria: si el primero prolongaba medidas clásicas de los últimos años, como la congelación de retribuciones o los límites en el aumento de efectivos, añadía una novedad, la fijación de una jornada laboral homogénea para el sector público. Por su parte, sería la norma de 2012 la que verdaderamente se haría acreedora de la calificación, al afectar a ámbitos como la supresión de una paga extraordinaria, la reducción de permisos y licencias, así como las retribuciones en caso de incapacidad temporal y los permisos sindicales. La denominación de "círculos de vulnerabilidad" que el profesor Jiménez Asensio empleó en 2011 para referirse al personal laboral y, especialmente al laboral no permanente, revelaría su pertinencia a la luz de la última reforma laboral, plasmada en la Ley 3/2012, de 6 de julio, que añadía dos nuevas disposiciones adicionales al Estatuto de los Trabajadores, la $20^{\mathrm{a}}$ y la $21^{\mathrm{a}}$, que despejó definitivamente las dudas acerca de la aplicación del despido por causas económicas, técnicas, organizativas o de producción al personal laboral del sector público, incluso con exclusión de otras medidas menos traumáticas como la suspensión temporal de la relación laboral. La opción por la reducción de efectivos parece, así, clara. Eso sí, sin más criterios que el tipo de relación jurídica que las sustenta. 
este trabajo contempla una doble aproximación: macro, a través de la elaboración de datos secundarios obrantes en los ámbitos estatales (Ministerio de Hacienda y Administraciones Públicas, MHAP, y sus bases de datos online y en especial el boletín estadístico del Registro de Personal) y mediante una aproximación meso a un objeto menor, los municipios que conforman el Área Metropolitana de Valencia (AMV), a través del estudio de los datos obrantes en los presupuestos y plantillas publicados en los boletines oficiales de la provincia de Valencia entre los años 2005 y 2012. Al respecto pensamos, con Salvador (2011:301) que estudiando la composición -y la evolución- de los diversos contingentes de profesionales que prestan servicio en los ayuntamientos, es posible apuntar impactos en las dinámicas de funcionamiento.

El modelo explicativo adopta los planteamientos encuadrados en la versión evolutiva del institucionalismo de la elección racional (IER) y del institucionalismo histórico (IH). Las instituciones son concebidas como "reglas del juego", formales e informales (Steinmo, 2013:137) las cuales dan forma a la interacción humana (North, 1993:13). Tales reglas son las que definen quién tienen derecho a tomar decisiones en cada ámbito, conformando un espacio social, una arena, cuyos elementos definitorios son los participantes y las situaciones de acción que estos deben encarar, en un planteamiento deudor del modelo de Ostrom y su denominado marco de Análisis y Desarrollo Institucional (ADI) (2013:44; 2011:109). Los fenómenos sociales son explicados a partir de su consideración como el resultado de múltiples procesos de decisión (Coleman, 2011:44). El modelo utilizado constituye un planteamiento fértil para efectuar tanto investigación diagnóstica como prescriptiva, sobre la base de considerar el EPL como una realidad compuesta por miles de ayuntamientos ${ }^{3}$ y en donde tienen lugar decisiones de política de personal a partir de la interacción entre los actores clave (Longo, 2000) de esta arena específica: cargos electos (con responsabilidad ejecutiva y de oposición), los partidos que los sustentan, altos funcionarios y directivos, así como representantes de los empleados públicos, en espacios políticos formales como los plenos municipales, las juntas de gobierno local o las mesas de negociación sindical, y también informales. Resulta imprescindible analizar el concreto entorno institucional y los incentivos que ofrece a los diversos agentes si se quiere realmente comprender el funcionamiento a escala "bottom up", en una visión abajo-arriba de la gestión pública, la premisa desde nuestro punto de vista para implementar reformas políticamente viables. Macro (tendencias) y micro (actores estrategas) se combinan así en un modelo capaz de proporcionar explicaciones plausibles.

A partir de este planteamiento, el resto del artículo busca en primer lugar, dar cuenta de cuál era la situación estructural del EPL en el año 2008, para seguidamente analizar los datos agregados existentes de los últimos cinco años, de 2009 a 2012. Por último se desciende un peldaño en el análisis para estudiar en una escala inferior -la metropolitana- las decisiones adoptadas por los ayuntamientos de un ámbito geográfico acotado.

\section{PERSPECTIVA MACRO: LAS POLÍTICAS DE RECURSOS HUMANOS EN LOS MUNICIPIOS ESPAÑOLES TRAS LA CRISIS}

¿Cuál es el contexto sobre el que operan tanto la crisis como las medidas de empleo público adoptadas? Una metáfora utilizada para describir la situación estructural del EPL hasta el año 2008 ha sido la de la "burbuja" (Cantero, 2012:159). Longo (2010), emplea el término para referirse no tanto al número de efectivos como a los "servicios públicos". Para calibrar este aspecto debe tenerse en cuenta que cuando en abril de 1979 se democratizan nuestros consistorios, estos tuvieron que hacer frente a unos desafíos urbanísticos y de dotación de equipamientos básicos palmarios, por no hablar de las carencias en materia de salud pública o en educación primaria. Con esos mimbres el desarrollo fue el previsible: los ayuntamientos se convirtieron en auténticos motores del cambio social, dando lugar a una de nuestras historias de éxito en democracia. Los municipios -en especial los urbanos- del siglo XXI en nada se parecen a los de finales de la década de los setenta. Este fenómeno se ha llegado a calificar como la "transformación urbana" de nuestro país (Iglesias, 2011:390).

Ciertamente la evolución de los efectivos ha sido notable, si se atiende a la tabla 1. Hay que tener en cuenta que alrededor de doscientos mil personas conformaban el total de empleados públicos al servicio de los ayuntamientos durante el tardofranquismo, según el estudio realizado en el entonces Instituto de Estudios de la Administración Local (IEAL) por Rodríguez Socorro (1975). Si se compara esta magnitud con la existente en 1984 -241.726- se constata cómo la evolución fue prudente en esos primeros años. No sería sino a partir de la década de los noventa y especialmente en la primera del 2000 cuando el crecimiento podría ser calificado con propiedad como "de burbuja", al doblar en poco más de veinte años el total de efectivos.

3 Hay que tener presente que en nuestro municipalismo ha arraigado una regla: la de que cada ayuntamiento es una Administración con notable autonomía a la hora de reclutar y retribuir al personal y en donde tras la Constitución de 1978 la supervisión previa existente en estas materias, desde 1981, fue suprimida. 
TABLA 1

EVOLUCIÓN DE LOS EFECTIVOS EN LOS AYUNTAMIENTOS ESPAÑOLES ANTES DE LA CRISIS

\begin{tabular}{|r|r|r|r|r|r|r|r|}
\hline \multicolumn{1}{|c|}{1984} & 1990 & 1996 & 2000 & 2002 & \multicolumn{1}{c|}{2006} & \multicolumn{1}{c|}{2008} \\
\cline { 2 - 8 } & & & & & & & \\
Total efectivos & 241.726 & 327.362 & 425.156 & 456.368 & 477.945 & 493.505 & 540.847 \\
(\% s/total AAPP) & $(15 \%)$ & $(15.4 \%)$ & $(20.5 \%)$ & $(19.3 \%)$ & $(20.7 \%)$ & $(20.2 \%)$ & $(20.9 \%)$ \\
\hline
\end{tabular}

FUENTE: elaboración propia sobre Carrillo Barroso (1991) y Boletín estadístico del Registro de Personal.

¿Qué explica este crecimiento tan notorio en unos años en los que se supone que las carencias más evidentes habían sido superadas? En otro lugar se ha ensayado una explicación (Cuenca, 2013). Una mezcla de factores políticos, institucionales y económicos lo hicieron posible. La segunda mitad de los ochenta contempló un conjunto de reformas que fueron aprovechadas por los actores que operaban en el sistema, en especial los partidos políticos. Reformas laborales y de la función pública, así como del sistema de gobierno local, facilitaron la incorporación masiva de personal incorporado con muy pocas garantías, algo que resultaba funcional para unos partidos emergentes, y que reproducirían con el tiempo una de las patologías típicas de nuestro municipalismo - incorporación precaria y estabilización posterior prácticamente restringida - favorecida ahora por un nuevo actor, el sindicato, que pasó a desempeñar un rol relevante en materia de negociación colectiva. Una coyuntura que además, se caracterizó por la llegada masiva de fondos provenientes de la Unión Europea.

Esta tendencia incremental se confirma si se analizan los gastos de personal de los ayuntamientos españoles a partir de 2002 (primera referencia disponible), en lo que supone más de un setenta por cien de aumento en el periodo (siete mil millones de euros), a razón de una tasa de crecimiento medio anual del 6.3\%. No será hasta 2010 -cuando los gastos de personal de los municipios españoles suponían ya dieciocho mil millones de euros-, cuando se interrumpa la dinámica incremental de este capítulo. El periodo comprendido entre ese año y 2012 muestra por vez primera una reducción significativa, del $6.6 \%$.

TABLA 2

DINÁMICA DE LOS GASTOS DE PERSONAL DE LOS AYUNTAMIENTOS ESPAÑOLES ANTES Y DESPUÉS DE LA CRISIS (EN MILES DE EUROS)

\begin{tabular}{|l|c|c|}
\cline { 2 - 3 } \multicolumn{1}{c|}{} & Periodo 2002-2010 & Periodo 2010-2012 \\
\hline Evolución del Capítulo I & +7.668 .553 & -1.196 .274 \\
& $(+73.6 \%)$ & $(-6.6 \%)$ \\
\hline Evolución total presupuestos municipales & +18.730 .586 & -7.038 .877 \\
& $(+52.8 \%)$ & $(-13 \%)$ \\
\hline
\end{tabular}

FUENTE: elaboración propia sobre datos de la Oficina virtual para la coordinación financiera con las Entidades Locales.

En ese mismo periodo de tiempo, los presupuestos municipales han pasado de casi treinta cinco mil quinientos millones de euros de 2002 a los cuarenta y siete mil del ejercicio 2010, lo que supone que los gastos de personal aumentan a mayor ritmo que los presupuestos en este periodo. No sería hasta los años 2009 y 2010 cuando los ayuntamientos redujeron sus presupuestos (el $1.8 \%$ y el $5 \%$ respectivamente), en un contexto en el que, no obstante, los gastos de personal seguían creciendo. Es destacable la reducción de los dos años siguientes, donde el capítulo I disminuirá casi mil doscientos millones de euros.

La senda de ajuste emprendida a partir de esta fecha se confirma si se analiza la evolución agregada de las plantillas municipales, tal y como muestra la tabla 3. 
TABLA 3

EVOLUCIÓN DE LOS EFECTIVOS DE LOS AYUNTAMIENTOS ESPAÑOLES DURANTE LA CRISIS

\begin{tabular}{l|r|r|r|r|r|r|}
\hline & \multicolumn{1}{c|}{2009} & \multicolumn{1}{c|}{2010} & \multicolumn{1}{c|}{2011} & \multicolumn{1}{c|}{2012} & \multicolumn{1}{c|}{2013} & \multicolumn{1}{c|}{$\begin{array}{c}\text { Diferencia en el } \\
\text { periodo }\end{array}$} \\
\cline { 2 - 7 } Total efectivos & 549.442 & 580.869 & 569.948 & 526.248 & 486.641 & -62.801 \\
(\% s/total AAPP) & $(20.8 \%)$ & $(21.5 \%)$ & $(21.1 \%)$ & $19.6 \%)$ & $(18.9 \%)$ & $(-11.4 \%)$ \\
\hline Personal Funcionario & 182.784 & 185.035 & 189.739 & 190.169 & 188.550 & +5.766 \\
(\% s/total EPL) & $(33.2 \%)$ & $(31.8 \%)$ & $(33.3 \%)$ & $(36.1 \%)$ & $(38.7 \%)$ & $(+3.1 \%)$ \\
\hline Personal laboral & 331.443 & 360.731 & 346.577 & 304.005 & 268.197 & -63.246 \\
(\% s/total EPL) & $(60.3 \%)$ & $(62.1 \%)$ & $(60.8 \%)$ & $(57.8 \%)$ & $(55.1 \%)$ & $(-19.1 \%)$ \\
\hline
\end{tabular}

FUENTE: elaboración propia sobre datos del Boletín estadístico del Registro de Personal.

De su análisis cabe destacar algunas notas. En primer lugar, que tal y como mostraron los datos presupuestarios, no fue hasta el año 2011 cuando se rompe la tendencia incremental, dos años después del inicio de la crisis. Puede apuntarse como hipótesis que el "pistoletazo de salida" en el ajuste del EPL no se dio hasta que tuvieron lugar las elecciones municipales de junio de ese año. Comportamiento acorde con los rasgos de política clientelar que aqueja a este nivel de gobierno (Cuenca, 2013).

Así las cosas, será a la altura de enero de 2013 cuando se refleje una reducción notable de las plantillas, más de sesenta mil efectivos, un $11 \%$ del total. Ahora bien, el comportamiento es notoriamente diferente en los diferentes colectivos. Si bien el personal laboral es el colectivo damnificado en la reducción, al suponer una quinta parte del colectivo total, sorprendentemente, aumenta levemente el porcentaje de empleados públicos sometidos a relación administrativa, tres puntos al final del periodo, en lo que es ni más ni menos que una respuesta racional -desde la perspectiva de algunos actores - a la circunstancia de que los ajustes solamente toman en cuenta el vínculo jurídico, lo que confirmaría que serían los "círculos de vulnerabilidad" a los que se ha hecho referencia, los únicos afectados en la reducción de plantillas, normalmente compuestos por empleados públicos más jóvenes y, en ocasiones, de mayor cualificación.

De modo complementario, hay que dejar constancia de que la reducción de personal laboral, fijo y no permanente, fue precedida por el colectivo que el boletín estadístico del registro central de personal engloba bajo el epígrafe de "otro personal" y que incluye tanto a personal eventual como a funcionariado interino. Este tipo de personal ha experimentado una contracción mayor.

TABLA 4

EVOLUCIÓN DEL COLECTIVO “OTRO PERSONAL” DURANTE LA CRISIS

\begin{tabular}{|l|r|r|r|}
\cline { 2 - 4 } \multicolumn{2}{c|}{2009} & 2013 & \multicolumn{2}{c|}{ Diferencia en el periodo } \\
\hline Colectivo de “otro personal” & 35.215 & & -5.321 \\
& & 29.894 & $(-15.1 \%)$ \\
\hline
\end{tabular}

FUENTE: elaboración propia a partir de datos del Registro de Personal.

Así las cosas, el análisis revelaría la estrategia de la reducción de plantillas seguida, de menor a mayor protección jurídica: eventuales -personal designado políticamente- seguidos de interinos y laborales temporales y finalmente, laborales fijos ${ }^{4}$.

\section{PERSPECTIVA MICRO: LOS MUNICIPIOS DEL ÁREA METROPOLITANA DE VALENCIA}

El Empleo Público Local (EPL) como institución engloba diversas realidades. Tantas como municipios. Pero hay una característica que conviene tener presente, y es la de que el EPL es una realidad principalmente urbana. España es un país de ciudades, y cuando se analiza la realidad de la gestión pública de personal de los 8.116 municipios, este dato debe tenerse presente en el análisis. La tabla 5 , que muestra solamente los municipios mayores de veinte mil habitantes refleja esta concentración poblacional.

4 Otra reducción significativa hay que consignar en ese periodo, la de los cargos municipales con dedicación exclusiva, que pasaron de casi once mil en 2008 a poco más de ocho mil quinientos en 2011, según datos proporcionados por la Tesorería General de la Seguridad Social con ocasión de la tesis doctoral de uno de los autores del artículo.

GESTIÓN Y ANÁLISIS DE POLÍTICAS PÚBLICAS, Nueva Época, nº 10 julio-diciembre 2013 ISSN: 1989-8991 - DOI: 10.24965/gapp.voi10.10117 
TABLA 5

ESTRUCTURA DEL MAPA MUNICIPAL EN ESPAÑA

\begin{tabular}{|l|c|c|c|}
\hline Número de habitantes & Número municipios & Habitantes que comprende & \% s total población española \\
\hline $20.000-50.000$ & 250 & 7.422 .185 & $15.8 \%$ \\
\hline $50.001-100.000$ & 82 & 5.857 .902 & $12.5 \%$ \\
\hline $100.001-500.000$ & 56 & 11.014 .339 & $23.5 \%$ \\
\hline 500.000 en adelante & 6 & 7.539 .304 & $16.1 \%$ \\
\hline TOTAL & 394 & 31.833 .730 & $67.9 \%$ \\
\hline
\end{tabular}

FUENTE: elaboración propia sobre datos del Censo de Población y Viviendas 2011.

Como puede observarse, 394 municipios mayores de veinte mil habitantes, menos del $5 \%$ del total, engloban a más de dos tercios de la población, cifrada en 47.129 .783 en enero de $2013^{5}$. Más ajustadamente, la mitad de la población vive en 144 ciudades que superan los cincuenta mil habitantes y que representan menos del dos por cien de nuestro mapa municipal, un dato a considerar cuando se diseñan reformas institucionales para este nivel de gobierno.

Dado que el EPL es una institución destacadamente urbana, hay que añadir otra nota característica, la de su notable metropolitanización (Tomás y Martí-Costa, 2011:37). Rasgo que engloba tanto un proceso social, la movilidad en un entorno cercano, como un fenómeno administrativo, la gestión concertada de una serie de políticas "hard" en ámbitos como el transporte público y el medio ambiente, pero que en nuestro país ha tenido una débil respuesta de su sistema político. Como es sabido, nuestro "Régimen Local” se caracteriza por su uniformidad, excepción hecha de la reforma de 2003, que otorgó carta de naturaleza, en forma de cierta diferenciación organizativa a las "grandes ciudades"6.

¿Qué es un "área metropolitana” y cuáles son sus rasgos definitorios? Si bien no existen definiciones canónicas y la delimitación es algo abierta, Tomàs (2011:285) la define como un área urbana polinuclear que conforma un mercado unitario de residencia y trabajo. Más específicamente, Sorribes (2012:244) enumera una serie de características: es una noción referida a la ciudad "real”, más allá de delimitaciones administrativas, compuesta por diversos núcleos poblacionales relevantes, con flujos constatables en el espacio (los de residencia, trabajo y consumo) y a los efectos que aquí interesan, generadores de diversas externalidades, algunas negativas, en especial en la gestión de servicios públicos. Circunstancia que es fuente de notables ineficiencias. En palabras de este último autor, "demasiadas como para no plantear ámbitos de gobierno supramunicipales, que son la base de una sensata profesionalización de la función pública local” (2012:328).

El área metropolitana de Valencia (AMV) puede calificarse como típica, en tanto existen políticas metropolitanas "clásicas", como el agua o los residuos sólidos urbanos, heredados de sus antecedentes organizativos (la Corporación Administrativa Gran Valencia de 1949 y el Consell Metropolità de l'Horta de 1986, posteriormente suprimido) aunque con carencias en los ámbitos de la planificación territorial y el medio ambiente, el transporte o las políticas sociales. Se trata de un gran núcleo de población, Valencia, con casi ochocientos mil habitantes, que da lugar a una "capital no consciente de serlo"(Alcalá-Santaella, 2011:201). Una capital, política y administrativamente limitada a su espacio geográfico, prácticamente con continuidad espacial (una conurbación en términos prácticos) de diversos núcleos poblacionales, algunos de ellos con poblaciones superiores a los 50.000 habitantes (Torrent, Paterna, Sagunto), pero la mayoría calificables como de pequeñas ciudades. La tabla 6 muestra la configuración municipal del AMV.

5 Cifras oficiales de la revisión del padrón municipal, aprobado por el Real Decreto 1016/2013, de 20 de diciembre.

6 Fue la Ley 57/2003, de 16 de diciembre, la que entre otros aspectos, añadió un nuevo Título, el X, a la Ley de Bases de Régimen Local de 1985, e introdujo ciertos elementos diferenciales de gestión a lo que se denominó como "municipios de gran población”, en forma de una mayor "ejecutividad" del gobierno municipal, por la vía de otorgar competencias propias, a la partir de entonces denominada Junta de Gobierno Local, en detrimento del Pleno. Hasta entonces, excepción hecha de Barcelona, la única singularidad de relevancia en el régimen local iba referida a los municipios regidos por el sistema de "concejo abierto". 
TABLA 6

DISTRIBUCIÓN DE LOS MUNICIPIOS ESTUDIADOS

\begin{tabular}{|l|c|c|}
\hline \multicolumn{1}{|c|}{ Población } & Número municipios & Porcentaje \\
\hline Hasta 5.000 habitantes & 17 & $26 \%$ \\
\hline 5.001-20.000 habitantes & 29 & $44 \%$ \\
\hline 20.001-50.000 habitantes & 16 & $24 \%$ \\
\hline 50.000 en adelante & 4 & $6 \%$ \\
\hline TOTAL & 66 & $100 \%$ \\
\hline
\end{tabular}

FUENTE: elaboración propia a partir del Censo de Población y Viviendas 2011.

En el proyecto de investigación en el que se inserta esta comunicación, se ha considerado que el AMV está compuesta por 76 municipios. El estudio ha obtenido datos de 66 de ellos, tomando como fuente principal la publicación de los presupuestos anuales y la plantilla anexa. Conviene dejar constancia de las limitaciones de esta fuente secundaria de datos. En primer lugar, un número sensible de ayuntamientos no han procedido a la aprobación de sus presupuestos, hallándose en situación de prórroga. Todos ellos muestran una notable disparidad de criterios en la confección de sus plantillas (de clasificación en Grupos, de denominaciones de plazas, etc.). No obstante, el análisis se ha centrado en tres temas, similares a los estudiados en el ámbito estatal: A) En la evolución de los gastos de personal. B) En la evolución cualitativa y cuantitativa de las plantillas. Y finalmente, C) en la evolución del grado de laboralización de las mismas. Todos ellos muestran tendencias similares a la constatada en el ámbito nacional, si bien con algunos elementos diferenciales. Siendo el objeto del estudio la estrategia adoptada tras la crisis, se han adoptado dos periodos para determinarla: el previo (2005-2008) y el posterior, si bien este último en dos subperiodos, habida cuenta de la limitación de datos, 2009-2011 y 2009-2012. Los ayuntamientos, además se ha desagregado por volumen poblacional.

El periodo inmediatamente anterior al estallido de la crisis financiera muestra la magnitud en el incremento de las plantillas.

TABLA 7

EVOLUCIÓN DE LAS PRINCIPALES MAGNITUDES 2005-2008 EN LOS AYUNTAMIENTOS DEL AMV

Hasta 5.000 habitantes $(\mathrm{n}=13)$

5.001-20.000 habitantes $(\mathrm{n}=24)$

20.001-50.000 habitantes $(n=12)$

50.000 en adelante $(n=4)$

$\mathrm{N}=53$

\begin{tabular}{|c|c|c|}
\hline Evolución gastos de personal & Evolución de los efectivos & Evolución del personal laboral \\
\hline$+3.5 \%$ & $+34 \%$ & $-1 \%$ \\
\hline$-0.6 \%$ & $+24 \%$ & $-3 \%$ \\
\hline$+1 \%$ & $+18 \%$ & $+1.5 \%$ \\
\hline$-5 \%$ & $+15 \%$ & $+1.5 \%$ \\
\hline$-\mathbf{1 . 1 . 1} \%$ & $+\mathbf{2 2 . 7 5} \%$ & $-1 \%$ \\
\hline
\end{tabular}

FUENTE: Elaboración propia a partir datos publicados en el BOPV.

Los efectivos totales aumentan casi en una cuarta parte en cuatro años, en una tendencia similar a la estatal. No obstante, se reflejan otros datos de interés: los gastos de personal no crecen al mismo ritmo (sin duda reflejo del incremento de los presupuestos municipales) y, destacadamente, el incremento de plantillas no afecta prácticamente al personal laboral. Su proporción, sensiblemente inferior a la media estatal, no se verá aumentada en el periodo, aunque a este respecto (como la del incremento total de empleados públicos) cabe afinar el análisis por tamaño del ayuntamiento. En los más pequeños, siempre será mayor la laboralización.

La tabla 8 muestra la evolución tras el crack de 20087, por lo que los presupuestos estudiados son los de 2009 en adelante, en dos periodos: hasta 2011 y en 2012.

7 La fecha convencionalmente manejada como de conciencia de la crisis, la de la quiebra de la firma Lehmann Brothers, en septiembre de 2008 . 
TABLA 8

\section{EVOLUCIÓN DE LAS PRINCIPALES MAGNITUDES DE LOS AYUNTAMIENTOS DEL AMV TRAS LA CRISIS}

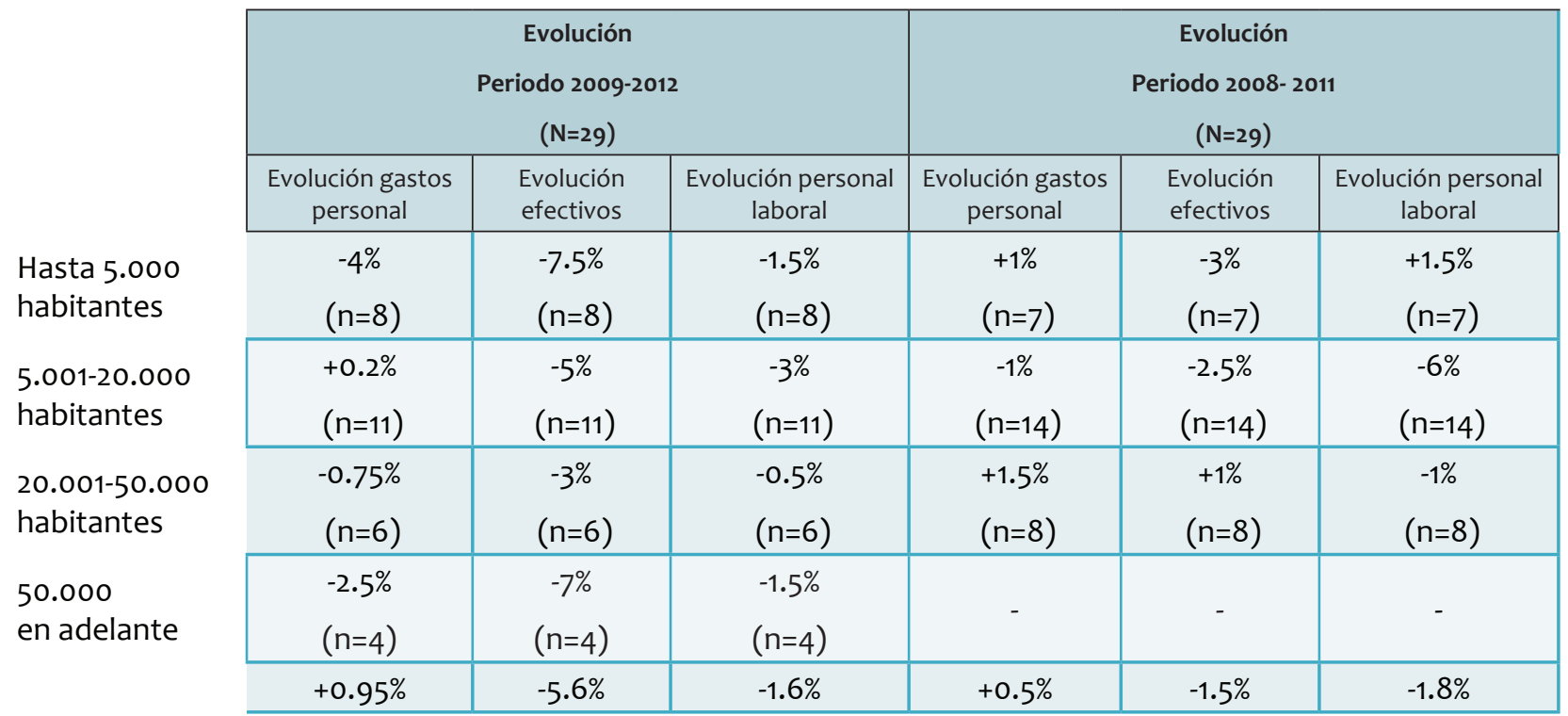

FUENTE: Elaboración propia a partir datos publicados en el BOPV.

La tabla refleja cómo, de modo paralelo a lo ocurrido en el ámbito estatal, los ayuntamientos valencianos pospusieron sus ajustes al año 2011, manifestándose sus efectos en los presupuestos posteriores, los correspondientes al ejercicio 2012. Los presupuestos de personal prácticamente se mantienen y empiezan a entreverse recortes de plantilla, aunque moderados (un 1.5\%), centrados principalmente en el personal laboral. Ahora bien, si se incorporan los datos correspondientes al año 2012 la magnitud aumenta sensiblemente: los ayuntamientos han disminuido sus plantillas más de un $5 \%$, ajuste que se realiza con dos datos significativos: en primer lugar, sin prácticamente disminución de los gastos de personal y sin que se proyecten en esta ocasión especialmente sobre el personal laboral.

Son magnitudes, en todo caso, inferiores al grado de contracción de plantillas y de gastos experimentado a escala nacional. ¿Cómo interpretar esta circunstancia? En primer lugar, cabría tener presente que los presupuestos se han contraído, pero la partida de personal es, de todas las dedicadas al gasto corriente, la más inelástica. Pese a la disminución de los presupuestos, los gastos de personal no disminuyen al mismo ritmo. Más interés revela la disparidad en la disminución de las plantillas (5.6\% del AMV frente al 11\% del total del EPL) y que a nuestro juicio no es tal, ya que en el caso del AMV los recortes lo son en plazas “de plantilla”, lo que significa que se trata de amortizaciones, normalmente de plazas ocupadas pero no cubiertas de modo permanente, bien por funcionarios de carrera o por laborales fijos. Hay que tener presente que los datos del registro de personal se obtienen, en lo que a la Administración Local respecta, de la Tesorería General de la Seguridad Social, que refleja los efectivos que

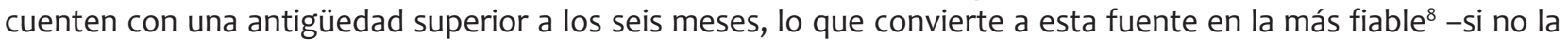
única- para estimar la magnitud de la disminución de las plantillas de los ayuntamientos. En todo caso, la tabla 8 muestra, en primer lugar, que no se han amortizado de modo significativo plazas de "laborales fijos" (las únicas susceptibles de ello) y que el grado de "funcionarización" de las plantillas de los municipios del AMV (más de dos tercios del total) es notable, tal y como muestra la tabla 9.

8 Al menos hasta que se disponga de los datos elaborados a partir de la Orden HAP/2105/2012, de 1 de octubre, por la que se desarrollan las obligaciones de suministro de información previstas en la ley de estabilidad presupuestaria de 2012, a tenor de la cual, en un hecho inédito hasta la fecha, las entidades locales están obligadas -artículo $7^{\circ}$ - a proporcionar al MHAP un volumen notable de datos referidos a su personal, tales como efectivos por clases, retribuciones satisfechas, etc. Esta modesta norma reglamentaria puede suponer un avance notable en la transparencia de las políticas públicas de recursos humanos. 
TABLA 9

EVOLUCIÓN DEL PERSONAL LABORAL DE LOS AYUNTAMIENTOS DEL AMV ANTES Y DESPUÉS DE LA CRISIS

\author{
Hasta 5.000 habitantes \\ 5.001-20.000 habitantes \\ 20.001-50.000 habitantes \\ 50.000 en adelante
}

\begin{tabular}{|c|c|}
\hline 2005 & $\mathbf{2 0 1 1}$ \\
\hline $52 \%$ & $62 \%$ \\
\hline $30 \%$ & $29.5 \%$ \\
\hline $32 \%$ & $28.5 \%$ \\
\hline $2 \%$ & $2.5 \%$ \\
\hline $29 \%$ & $30.6 \%$ \\
\hline
\end{tabular}

FUENTE: Elaboración propia a partir datos publicados en el BOPV.

En el año 2005 el porcentaje nacional de laboralización de las plantillas de las entidades locales, se hallaba en torno al 60\%. Los municipios del AMV mostraban un grado sensiblemente inferior, menos de la mitad del dato estatal, destacando al respecto los grandes municipios, con un grado insignificante de personal laboral. Este dato explicaría por qué una vez sobrevenida la crisis apenas hubo ajuste en las plantillas fijas y confirmaría cómo este se centró en las “capas externas" de los "círculos de vulnerabilidad”, los funcionarios interinos y los laborales temporales o "indefinidos", precedidos por los cargos de designación política, pero sin afectar de modo significativo al siguiente escalón, el de los laborales "fijos" o de plantilla".

Es decir, que ha habido ajuste, pero el mismo todavía no ha afectado significativamente a lo que podríamos denominar como el "núcleo duro" de las plantillas, y que tal reducción, en el caso de las plantillas del AMV, probablemente sea similar al operado a escala nacional, máxime si tenemos en cuenta la diversidad de fuentes (plantillas publicadas en boletines oficiales para el AMV, datos del Fichero General de Afiliación para el boletín del MHAP). Así las cosas, la estrategia de disminución sí habría seguido la pauta de basarse en la facilidad de extinción de la relación de servicio (eventuales, funcionarios interinos, laborales por tiempo determinado y laborales "indefinidos"). Esta interpretación se asienta si tenemos en cuenta que, en el AMV, apenas ha variado la composición cualitativa de las plantillas, antes y después de la crisis, tal y como refleja la tabla 10,

TABLA 10

EVOLUCIÓN CUALITATIVA DE LAS PLANTILLAS DE LOS AYUNTAMIENTOS DEL AMV ANTES Y DESPUÉS DE LA CRISIS

\begin{tabular}{|l|c|c|}
\cline { 2 - 3 } \multicolumn{1}{c|}{} & 2005 & 2011 \\
\hline Policía Local & $24 \%$ & $24 \%$ \\
\hline Servicios' Personales & $19 \%$ & $20 \%$ \\
\hline Personal de Oficios $^{2}$ & $27 \%$ & $28 \%$ \\
\hline Personal Burocrático $^{3}$ & $30 \%$ & $28 \%$ \\
\hline
\end{tabular}

FUENTE: Elaboración propia a partir datos publicados en el BOPV.

1 Este colectivo incluye personal de servicios sociales, cultura y fiestas, deportes, juventud, mujer, tercera edad, inmigración y promoción económica.

2 Este colectivo incluye efectivos dedicados a oficios como la jardinería, la mecánica, personal de obras y servicios, alumbrado y similares.

3 La rúbrica engloba personal dedicado a secretaría, intervención, rentas y recaudación, administración general, personal, contratación y similares.

9 Técnicamente hay que distinguir entre personal laboral "fijo" y personal laboral "indefinido". Como es sabido, en Derecho del Trabajo ambas denominaciones son intercambiables. Ahora bien, en el ámbito público, tal distinción sí resulta operativa, ya que con la denominación "indefinido", o literalmente, "Indefinido no fijo de plantilla", lo que se revela es una patología. La categoría jurídica "indefinido", ahora contemplada por las leyes (art. 8.2.c de la Ley 7/2007, de 12 de abril, del Estatuto Básico del Empleado Público o por la Disposición Adicional decimoquinta del Estatuto de los Trabajadores, introducida por la reforma de 2010, la Ley 35/2010, de 17 de septiembre), fue, en sus inicios, una creación jurisprudencial mediante la que se trataba de dar encaje a una realidad: la de que si en el ámbito público se utilizaban las respuestas que la legislación ordinaria preveía para el fraude de ley en la contratación temporal (tales como la falta de causa habilitante de la temporalidad o el encadenamiento de contratos con el mismo trabajador), la sanción de fijeza, (véase por todas, la sentencia de la Sala de lo Social del Tribunal Supremo, de 11 de febrero de 1991), traía como consecuencia el acceso a empleos públicos de trabajadores que no siempre se habían reclutado respetado los principios de mérito, capacidad y publicidad consagrados tanto en la Constitución (artículos 23 y 103) como en la legislación local (artículo 103 de la Ley 7/1985, de 2 de abril, reguladora de las Bases de Régimen Local). Así las cosas, será a partir de 1996 (sentencia de 7 de octubre de 1996, de la Sala de lo Social del Tribunal Supremo) y sobre todo, 1998, (sentencias de idéntico tribunal, en Sala General, de 20 y 21 de enero de 1998) y 2002 (sentencia de 27 de mayo) cuando se consolide este especie de "tertium genus" entre laborales temporales y fijos, con notables controversias jurídicas acerca de su tratamiento en materias como la extinción o el régimen indemnizatorio. Materialmente, este colectivo en nada se diferencia de un interino, un contratado a término, bien hasta que la plaza se cubra conforme a criterios de igualdad de oportunidades, o se amortice, tal y como recientemente ha dejado establecido la sentencia de la Sala de lo Social del Tribunal Supremo, de 22 de julio de 2013. 
Además de reflejar pequeñas variaciones en la evolución, y confirmarse que la facilidad jurídica de extinción ha sido el principal criterio de reducción de personal, la tabla permite formarse una idea de la composición de las plantillas municipales, mostrando así algunas pautas: notables esfuerzos en seguridad pública y un grado de burocratización destacado, calificativo pertinente si a este dato le añadimos el que solamente un veinte por cien de las plantillas se corresponden con plazas de carácter técnico (grupos A y B, según la legislación de empleo público) y, destacadamente, revela que el foco de la reforma local en ciernes (los denominados "servicios impropios" en gran parte) suponen una quinta parte de los efectivos, lo que puede sugerir un orden de magnitud a la hora de calcular la incidencia de la misma sobre el personal.

\section{CONCLUSIONES: LA SILENCIOSA REDUCCIÓN DE EMPLEO DE LOS AYUNTAMIENTOS ESPAÑOLES}

Por el volumen de efectivos afectados, los municipios de nuestro país han procedido a una suerte de regulación de empleo silente en apenas dos años. En 2011 cambiará, por primera vez, la tendencia incremental de los efectivos iniciada en los años ochenta. A diferencia de la Administración General del Estado y de las Comunidades Autónomas, que habían iniciado la reducción de personal con anterioridad. Previamente en 2010, los gastos de personal, iniciarán también su contención, que hasta esa fecha habían crecido anualmente en porcentajes superiores a los de los presupuestos.

Los datos obtenidos a escala estatal y del AMV confirman la hipótesis y darían respuesta a las preguntas de investigación: el vínculo jurídico ha sido el medio para ajustar a la baja los efectivos. El proceso, precedido por los cargos electos con dedicación exclusiva y los de designación política, se habría basado en la facilidad de supresión de la relación de servicio, en una prelación que pasaría primero por funcionarios interinos y laborales temporales y posteriormente laborales "indefinidos" y, por último, fijos. El orden de magnitud es relevante si se atiende al volumen: más de un diez por ciento de efectivos, un cinco por ciento de plazas "de plantilla". Tampoco han sido la materialización de un marco de reflexión estratégica. Los datos obtenidos de los municipios del AMV confirman que la reducción se ha proyectado sobre los "círculos de vulnerabilidad”, sin priorizar sectores de política pública o servicios públicos concretos y, por tanto, sin ningún tipo de previsión, que tenga en cuenta variables como la cualificación o la edad media, lo que hace presumir una elevada incidencia de estas prácticas sobre la composición futura de las plantillas. Se ha obviado una herramienta de gestión del empleo público, los planes de recursos humanos, que trató de potenciarse mediante el artículo 69 del Estatuto Básico del Empleado Público. Así las cosas, un aspecto a evaluar es el impacto que tendrá sobre una burocratizada y formalista gestión municipal de recursos humanos la supresión del colectivo que dota de cierta flexibilidad a las políticas de personal.

Por paradójico que resulte, los datos -estatales y del AMV- muestran que la estrategia seguida es perfectamente racional, ajustada a los intereses de los actores en presencia. Esto es, disminuye globalmente el personal, pero el análisis cualitativo revela algunas estrategias, Así, si bien el personal laboral disminuye, el funcionario aumenta. No obstante, en ámbitos altamente funcionarizados (como el AMV) dicha estrategia ha resultado de más difícil implementación. Así las cosas, los cargos públicos han primado estrategias cortoplacistas y de fácil adopción para hacer frente a la caída de ingresos fiscales. Los sindicatos han adoptado estrategias de blindaje de sus efectivos, aumentando la funcionarización. Adicionalmente hay que tener en cuenta que no se trata solamente de que se carezca de medios jurídicos de reordenación de la plantilla estable (la funcionarial señaladamente), sino que se carece de incentivos y capacidades administrativas (de planificación e innovación señaladamente) que posibiliten una estrategia alternativa. Más que de burbuja de efectivos, sería una burbuja de ineficacia gestora la que aqueja al empleo público local en nuestro país.

Finalmente, desde una perspectiva científico-social, disponemos de datos que avalan el planteamiento de que el funcionamiento del empleo público local en nuestro país es resultado del comportamiento de los agentes que en él operan, atentos a los incentivos y al contexto para alcanzar sus intereses. Un planteamiento a tener en cuenta cuando se proyectan estrategias de reforma institucional. 


\section{BIBLIOGRAFÍA}

AlCAlÁ-SAntAella, F. et. al. (2011): "Valencia”. En: IGlesias, M., Martí-Costa, M., Subirats, J. y TomÁs, M. Políticas Urbanas en España: grandes ciudades, actors y gobiernos locales. Pp. 201-227. Barcelona. Icària.

BECK, U. (2012): Una Europa alemana. Madrid. Paidós.

CANTERO, J. (2012): “El debate sobre la externalización y el número de funcionarios en nuestras Administraciones Públicas". En: FuentetAJA, J.A. y CANTERo, J. (dirs.): Crisis económica y Función Pública. Pp. 151-191. Madrid. Aranzadi.

CARRILLO, E. (1991): Gestión de recursos humanos, presupuestación y hacienda local en España. Madrid. Instituto de Estudios Fiscales.

COLEMAN, J. (2011 [e. o. 1990]): Fundamentos de teoría social. Madrid. CIS.

CUENCA, J. (2013): "El sistema de mérito en los gobiernos locales españoles: una institucionalización problemática”. En: Revista Vasca de Gestión de Personas y Organizaciones Públicas, № 4. Pp. 24-39.

FuentetAJA, J.A. (2012): “De la Función Pública en tiempos de crisis a la crisis de la Función Pública”. En: FuENTETAJA, J.A. y CANTERO, J. (dirs.): Crisis económica y Función Pública. Pp. 73-107. Madrid. Aranzadi.

GOLDTHORPE, J. (2010): De la sociología. Números, narrativas e integración de la investigación y la teoría. Madrid. CIS.

IGLESIAS, M. et. al. (2011): "La gran transformación urbana en España. Balance y conclusiones de las interrelaciones entre gobiernos locales y políticas urbanas en la democracia española". En: IGLESIAS, M., MARTí-COSTA, M., SUBIRATS, J. y TOMÁs, M. Políticas Urbanas en España: grandes ciudades, actores y gobiernos locales. Pp. 389-4037. Barcelona. Icària.

JIMÉNEZ ASENSIO, R.

- 2011: “Qué hacer? El empleo público local ante la crisis: políticas de ajuste ante un marco de reformas”. En: El empleo público local ante la crisis. Pp. 13-87. Granada. CEMCI-Fundación Democracia y Gobierno Local.

- 2012: "Debilidades del marco normativo estructural del empleo público para afrontar un escenario de profunda y prolongada crisis fiscal “.En: FUENTETAJA, J.A. y CANTERO, J. (dirs.): Crisis económica y Función Pública. Págs. 39-72. Madrid. Aranzadi

LAGO, I. (2008): La lógica de la explicación en las ciencias sociales. Una introducción metodológica. Madrid. Alianza.

LLOPIS, E. y MALUqUER DE Motes, J. (eds.) (2013): España en crisis. Las grandes depresiones económicas 13482012. Barcelona. Pasado y Presente.

LONGO, F.

- 2000: "Pensar lo público: políticos, directivos y sindicatos como actores de la gestión de recursos humanos en las Administraciones Públicas”. En: Pensar lo público. ZAFRA, M. y LONGO, F. (coords.). CEMCI. Granada. Pp. 473-512

- 2010: "La burbuja del servicio público". El Periódico de Catalunya. Edición del lunes 18 de octubre de 2010.

MÁRQUEZ CRUZ, G. (1997): “Transición y normalización del sistema político local en España.” En: ALBA y VANACLOCHA (coord.): El sistema político local: un nuevo escenario de gobierno. Pp. 141-204. Madrid. Universidad Carlos III y BOE.

NORTH, D. (1993): Instituciones, cambio institucional y desempeño económico. México, FCE.

OSTROM, E.

- (2013) [e. 0. 2005]: Comprender la diversidad institucional. Oviedo. KRK ediciones.

- (2011) [e. o. 1990]: El gobierno de los bienes comunes. La evolución de las instituciones de acción colectiva. México. Fondo de Cultura Económica. $2^{\mathrm{a}}$ ed.

Palomar Olmeda, P. (2012): “Las políticas de restricción del gasto público como elemento de ordenación de la gestión pública de recursos humanos y las formas de su articulación”. En: FUENTETAJA, J.A. y CANTERO, J. (dirs.): Crisis económica y Función Pública. Pp. 109-149. Madrid. Aranzadi.

PETERS, G. (2003): El nuevo institucionalismo: la teoría institucional en ciencia política. Barcelona. Gedisa.

GESTIÓN Y ANÁLISIS DE POLÍTICAS PÚBLICAS, Nueva Época, no 10 julio-diciembre 2013 ISSN: 1989-8991 - DOI: 10.24965/gapp.voi10.10117 
Pierson, P. (2004): Politics in Time: History, Institutions and social analysis.

RAMıó, C. (2011): “La Administración que se espera para después de la crisis”. En: GIGAPP Estudios (WP-201102). Disponible en: www.gigapp.org (descargado el 11 de febrero de 2013).

SAlvador, M. (2011): "Disseny institucional: Organització I Ocupació Pública per al desenvolupament de polítiques públiques”. En: DíAz, L. (ed.) Polítiques Públiques dels municipis catalans. Barcelona. Fundació Pi i Sunyer.

SÁNCHEZ MoRÓN, M. (2012): “Ajustes y reformas en el empleo público: situación actual y perspectivas”. En: FuentetAjA, J.A. y CANTERo, J. (dirs.): Crisis económica y Función Pública. Pp. 21-37. Madrid. Aranzadi.

SORRIBES, J. (Dir.) (2012): La Ciudad: economía, espacio, sociedad y medio ambiente. Valencia. Tirant lo Blanch.

StEINMO, S. (2013): “Institucionalismo histórico”. En: DelLA PORTA, D. y KeATING, M. Enfoques y metodologías de las ciencias sociales. Pp. 131-151. Madrid. Akal.

StIGLITZ, J. (2012): El precio de la desigualdad. El 1\% de la población tiene lo que el 99\% necesita. Madrid. Taurus.

TOMÁs, M. (2011): “La escala metropolitana: un análisis transversal”. En: IGLESIAS, M., MARTí-COSTA, M., SUBIRATS, J. y TOMÁs, M. Políticas Urbanas en España: grandes ciudades, actors y gobiernos locales. Pp. 283-305. Barcelona. Icària.

TOMÁs, M. y MARTí-COSTA, M. (2011): “La reconfiguración de la Agenda Urbana: el debate europeo”. En: IGLESIAS, M., MARTí-COSTA, M., SUbIRATS, J. y TOMÁs, M. Políticas Urbanas en España: grandes ciudades, actors y gobiernos locales. Pp. 23-41. Barcelona. Icària. 